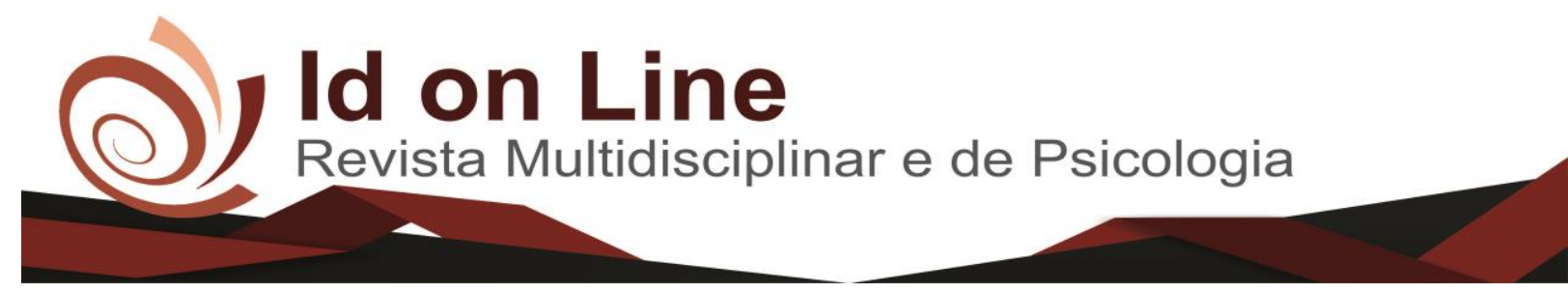

Artigo

\title{
Qualidade de vida e depressão entre pacientes carentes em tratamento oncológico na Casa do Amor em Vitória da Conquista BA
} Luciana Gonçalves Lima I; Stifanny Brenda Alves Dos Santos ${ }^{1}$; Gisele Norberto Mattos Gomes ${ }^{1}$; Leilane Trindade Gomes ${ }^{1}$;
Valéria Pires Freitas ${ }^{\text {}}$; Diego Andrade Caetité ${ }^{1}$; Stênio Fernando Pimentel Duarte ${ }^{123}$

\begin{abstract}
Resumo: A Oncologia está voltada para a forma como o câncer se evolui no organismo e qual o tratamento mais apropriado para cada tipo de neoplasia. De acordo com a Organização Mundial da Saúde (OMS), o câncer é a segunda causa de morte em países desenvolvidos, perdendo apenas para doenças cardiovasculares. Em termos estatísticos, podemos assegurar que o câncer é a doença crônica mais tratável no momento, cerca de $50 \%$ dos casos são curados. No Brasil estima-se que este número seja mais baixo, devido ao fato de que os diagnósticos são feitos tardiamente. As causas de câncer são diversas, podendo ser externas, internas ou inter-relacionadas. Mesmo depois dos progressos com o tratamento para o câncer e as chances de sobrevivência, cada vez maiores, o câncer ainda está correlacionado como uma doença que provoca dor, sofrimento e morte iminente (KUBLER-ROSS, 1981), fazendo com que assim o diagnostico tenha um grande impacto psicológico no individuo, resultando em ansiedade; angustia; duvidas; ódio; desesperança; medo entre outros. Segundo Minayo, Hartz e Buss (2000), "qualidade de vida tem sido ligada ao grau de satisfação encontrado na vida familiar, amorosa, social e ambiental e à própria estética existencial". Foi realizado um estudo transversal, quantitativo com o objetivo de avaliar a qualidade de vida e depressão dos pacientes oncológicos em tratamento na Casa do Amor em vitória da Conquista. Foram entrevistados 30 pacientes, 10 mulheres e 20 homens ambos diagnosticados câncer.
\end{abstract}

Palavras-Chave: Câncer. Depressão. Qualidade de vida

\section{Quality of life and depression among needy patients in cancer treatment at Casa do Amor in Vitoria da Conquista Bahia}

\begin{abstract}
Oncology is focused on how cancer evolves in the body and which treatment is most appropriate for each type of neoplasm. According to the World Health Organization (WHO), cancer is the second leading cause of death in developed countries, second only to cardiovascular disease. In statistical terms, we can ensure that cancer is the most treatable chronic disease at the moment, about 50\% of cases are cured. In Brazil it is estimated that this number is lower, due to the fact that the diagnoses are made late. The causes of cancer are diverse, and may be external, internal or interrelated. Even after the progress with the treatment for cancer and the chances of survival increasing, the cancer is still correlated to a disease that causes pain, suffering, and impending death (KUBLER-Ross, 1981), making just the diagnosis have a major psychological impact on the individual, resulting in anxiety; anguish; doubts; hate; hopelessness; fear among others. According to Minayo, Hartz and Buss (2000), "quality of life has been linked to the degree of satisfaction found in family, love, social and environmental life and to existential esthetics itself." A cross-sectional study, quantitative in order to assess the quality of life and depression of cancer patients undergoing treatment in the House of Conquest Victory in Love. We interviewed 30 patients, 10 women and 20 men both diagnosed cancer.
\end{abstract}

Keywords: Cancer. Depression. Quality of life 


\section{Introdução}

De acordo com a Organização Mundial da Saúde (OMS), o câncer é hoje a segunda causa de morte em países desenvolvidos, perdendo apenas para doenças cardiovasculares (SANTOS, BARTIRA DE GODOY MARANHÃO SANTOS et al., 2010). A doença representa a maior incidência mundial de tumores malignos, sendo o câncer de mama o que mais atinge a população feminina, o Brasil é o país com maior mortalidade de mulheres pelo câncer de mama (AZEVEDO SILVA; ROBERTO BORGES DE SOUZA-JÚNIOR; NOGUEIRA DAMACENA, 2017).

Se analisarmos em termos estatísticos, pode-se afirmar que o câncer é a doença crônica mais tratável no momento correspondendo a cerca de 50\% dos casos, considerando os países desenvolvidos (CELIS; PAVALKIS, 2017). No Brasil estima-se que este número seja menor, devido ao fato de que os diagnósticos são feitos muito tarde (INCA, 2017).

Segundo Rodrigues e colaboradores (2015) o Câncer é um conjunto de mais de 100 doenças tendo em comum o crescimento desordenado das células, que invadem algumas partes do corpo, gerando um tecido anormal formando um tumor. O corpo é formado por trilhões de células, as saudáveis vão se multiplicar para formar novas células, quando o organismo não necessita mais delas, elas morrem, o crescimento fora de controle e a invasão de outros tecidos é o que torna uma célula cancerosa (ALMEIDA, 2004, p. 1).

Mesmo após os avanços com o tratamento para o câncer e as chances de sobrevivência, cada vez maiores, o câncer ainda é relacionado com uma enfermidade que acarreta dor, sofrimento e morte iminente (KUBLER-ROSS, 1981). Tornando que tal diagnostico produza um grande impacto psicológico no individuo, resultando em ansiedade; angustia; duvidas; ódio; desesperança; medo entre outros (SALCI; SALES; MARCON, 2008).

Os indivíduos com câncer sofrem grande impacto emocional e psicossocial, por ser uma doença que carrega um significado muito forte, o da morte, transformando-se em um estigma para quem é acometido por ela (RAMOS, 2016). O portador de neoplasia maligna passa por um processo de várias perdas, que começa desde o diagnóstico, durante o tratamento e prognóstico, podendo prejudicar principalmente a qualidade de vida da pessoa (MINAYO, HARTZ E BUSS, 2000). 
Baseado no que foi explanado o presente trabalho teve como objetivo avaliar a qualidade de vida e depressão dos pacientes carentes em tratamento oncológico na casa do Amor de Vitoria da Conquista- Ba.

\section{Metodologia}

Foi realizado um estudo transversal, quantitativo, com o intuito de avaliar a qualidade de vida e depressão dos pacientes carentes oncológicos em tratamento na Casa do Amor, uma instituição filantrópica que atende pacientes oncológicos de toda a região da Bahia, e norte de Minas Gerais. Os mesmos são assistidos nas diversas fases do tratamento.

A pesquisa foi realizada nos dias 04 e 05 de Outubro de 2017 na Casa do Amor localizada na cidade de Vitória da Conquista- BA, foram entrevistados 30 pacientes oncológicos, sendo 10 mulheres de idade entre 43 e 86 anos e 20 homens entre 44 e 75 anos, foi aplicado um questionário EORTC QLQ-C30 e um termo de consentimento assinado pelos pacientes.

\section{Resultados e discussões}

Foram entrevistados 30 pacientes oncológicos, 10 mulheres $(33,33 \%)$ de idade entre 43 e 86 anos, sendo 9 com diagnóstico de câncer de mama e 1 câncer de estômago e 20 homens (66,67\%), entre 44 e 75 anos, com diagnósticos de vários tipos de câncer, prevalecendo o câncer de próstata, nos homens a incidência maior é o câncer de próstata onde atinge $40 \%$ (n=8) dos pacientes entrevistados, seguido por câncer de esôfago $20 \%(n=4)$, câncer de estômago e de pulmão, cada um com 10\% (n=2) câncer de perna, garganta, língua e reto, 5\% (n=1) dos pacientes.Foi feita uma análise sobre a qualidade de vida e depressão dos pacientes após a doença.

Com base na tabela 1 percebe-se que à medida que a qualidade de vida da paciente aumenta, a depressão diminui. Pois podemos observar que pacientes que apresentam uma qualidade de vida como boa, não vão apresentar sintomas de depressão ou os sintomas são 
poucos. Já pacientes que apresentam uma qualidade de vida como regular a maior parte delas apresentam sintomas de depressão. A paciente que apresentou uma qualidade de vida como ruim apresentou sintomas de depressão.

Tabela 1: Depressão e qualidade de vida em pacientes com câncer em tratamento na casa do Amor no município de Vitoria da Conquista- BA.

\begin{tabular}{llcccc}
\hline Sexo & \multicolumn{5}{c}{ Qualidade de Vida } \\
\hline Feminino & & Ruim & Regular & Boa & Total \\
& Sem Depressão & 0 & 0 & 4 & $4(40 \%)$ \\
& Com Depressão & 0 & 1 & 5 & $6(60 \%)$ \\
\hline \multirow{2}{*}{ Masculino } & Sem Depressão & 1 & 2 & 12 & $15(78,9 \%)$ \\
& Com Depressão & 1 & 3 & 0 & $4(21,1 \%)$ \\
\hline & Total Geral & 2 & 6 & 21 & $29(100 \%)$ \\
\hline
\end{tabular}

Fonte: Pesquisa dos autores

Eles não apresentam uma relação entre os sintomas de depressão com a qualidade de vida. Observa-se que o fato de apresentar uma baixa qualidade de vida não quer dizer que apresente sintomas de depressão, já que a maioria deles disseram não apresentar sintomas de depressão, e os que responderam apresentar algum sintoma não apresentavam uma baixa qualidade de vida. Então 65\% $(n=13)$ dos homens entrevistados classificaram a sua qualidade de vida como boa, $20 \%(\mathrm{n}=4)$ dos pacientes classificaram como regular $10 \% \quad(\mathrm{n}=2)$ classificaram como ruim, e $5 \%(n=1)$ não responderam quando perguntados sobre a sua qualidade de vida.

Esses dados são divergentes em parte dos encontrados em Silva e colaboradores em 2017 que verificou uma associação entre a qualidade de vida e alterações cognitivas em uma revisão sistemática sobre associação entre ansiedade, depressão e qualidade de vida em pacientes oncológicos. Os nossos dados foram opostos também dos dados obtidos por Ponte no ano de 2016 na associação entre pacientes com tumores de pele e morbidade psicológica.

Quijada e colaboradores (2017), homens com diagnóstico inicial obtido pelas alterações no exame de Antígeno Prostático Específico e estadiamento II, indicaram queda na qualidade de vida, logo não correlaciona com os resultados obtidos pela pesquisa.

De acordo com Ferreira e colaboradores (2017) a qualidade de vida em mulheres acometidas por câncer de mama, demonstram que o Estado Geral de Saúde atingiu uma média 
de 73,1, então podemos considerar que as pacientes avaliam sua Qualidade de Vida como satisfatória corroborando assim com os resultados analisados na pesquisa.

\section{Conclusão}

Os resultados obtidos demonstraram que todos os pacientes entrevistados, a maioria considerava que sua qualidade de vida eram boas, e sobre a depressão dificilmente se sentiam deprimidos, pois o fato de estarem se tratando, era uma grande vitória, pois as chances de vida eram maiores, além de receber tratamento adequado, eram acolhidos como muito amor e respeito na Casa do Amor, pois se sentiam em casa. Pode perceber-se que a doença não era a morte para eles e sim uma doença crônica que pode ser tratada e controlada com tratamento e medicamentos adequados.

A qualidade de vida geralmente está associada com a depressão, ou seja, se você está deprimido, provavelmente sua qualidade de vida não estará boa, o que acaba afetando a vida desses pacientes em todos os sentidos.

\section{Referências}

ALMEIDA, Marcos Antonio Bettine de; GUTIERREZ, Gustavo Luis; MARQUES, Renato. Qualidade de vida: definição, conceitos e interfaces com outras áreas, de pesquisa. São Paulo: Escola de artes, ciências e humanidades-EACH/USP, p. 142, 2012.

AZEVEDO SILVA, G. I.; ROBERTO BORGES DE SOUZA-JÚNIOR, P. I.; NOGUEIRA DAMACENA, G. Detecção precoce do câncer de mama no Brasil: dados da Pesquisa Nacional de Saúde, 2013. Inquéritos Artigo Original Rev Saude Publica, v. 51, n. 1, 2017.

CELIS, J. E.; PAVALKIS, D. A Mission-oriented Approach to Cancer in Europe: A Joint Mission/Vision 2030. Journal of neurochemistry, v. 140, n. 6, p. 874-888, mar. 2017.

DOS SANTOS QUIJADA, Patrícia Daniela et al. Qualidade de vida relacionada à saúde de pacientes com câncer de próstata. Revista Cuidarte, v. 8, n. 3, p. 1826-38, 2017.

FERREIRA, Beatriz Machado Lança et al. AVALIAÇÃO DA QUALIDADE DE VIDA EM MULHERES ACOMETIDAS POR CÂNCER DE MAMA EM UMA UNIDADE PARTICULAR, NO MUNICÍPIO DE CAMPOS DOS GOYTACAZES, RJ. Biológicas \& Saúde, v. 7, n. 24, 2017. 
INCA. INCA - Ações e Programas - Vigilância do Câncer e seus Fatores de Risco. Disponível

em: <http://www2.inca.gov.br/wps/wcm/connect/acoes_programas/site/home/nobrasil/programa_ epidemiologia_vigilancia/>. Acesso em: 11 nov. 2017.

KUBLER-ROSS E. Domande e risposte sulla morte e il morire, it. vers. Como: Edizioni Red; 1981.

MINAYO, Maria Cecília de Souza et al. Qualidade de vida e saúde: um debate necessário. 2000.

RAMOS, V. A. B. O Papel Do Psicólogo Na Doença Oncológica E As Suas Fases. Psicologia.Pt, p. 1-10, 2016.

SALCI, M. A.; SALES, C. A.; MARCON, S. S. Sentimentos de mulheres frente ao cancer. @ BULLET Rev. enferm. UERJ, v. 17, n. 1, p. 4646-51, 2008.

SANTOS, BARTIRA DE GODOY MARANHÃO SANTOS, S. C. et al. Frequência de Realização do Autoexame das Mamas e Mamografia na Detecção de Nódulos em Mulheres de Baixa Renda na População Sul Fluminense. Revista de Saúde, v. 1, n. 1, p. 25-31, 2010.

SANTOS, BARTIRA DE GODOY MARANHÃO SANTOS, S. C. et al. Frequência de Realização do Autoexame das Mamas e Mamografia na Detecção de Nódulos em Mulheres de Baixa Renda na População Sul Fluminense. Revista de Saúde, v. 1, n. 1, p. 25-31, 2010.

SILVA, D. DA. Instrumentos de avaliação de ansiedade, depressão e qualidade de vida em pacientes pré/pós transplante de células-tronco hematopoiéticas: uma revisão sistemática. 2017.

WORLD CANCER RESEARCH FUND/AMERICAN INSTITUTE FOR CANCER RESEARCH. Food, nutrition, physical activity and prevention of cancer: A global perspective. Washington: American Institute for Cancer Research; 2007.

\section{Como citar este artigo (Formato ABNT):}

LIMA, Luciana G.; SANTOS, Stifanny B.A dos; GOMES, Gisele N.M.; GOMES, Leilane T.; FREITAS, Valéria P.; CAETITÉ, Diego A.; DUARTE, Stênio F.P. Qualidade de vida e depressão entre pacientes carentes em tratamento oncológico na Casa do Amor em Vitória da Conquista BA. Id on Line Revista ultidisciplinar e de Psicologia, 2017, vol.12, n.39, p.38-43. ISSN: 1981-1179.

Evolução Histórica do Cooperativismo no Setor Agropecuário

Recebido: 13.11 .2017

Aceito: 14.11 .2017 